\title{
PERSPECTIVA DEL DERECHO INTERNACIONAL DEL MEDIO AMBIENTE
}

(Primer semestre 2017)

\author{
SUSANA BORRÀs PENTINAT \\ Profesora contratada doctora de Derecho Internacional Público \\ Universitat Rovira i Virgili
}

[DOI: http://dx.doi.org/10.17345/rcda.1849] 1 
Sumario. 1. Las decisiones de $66^{\mathrm{a}}$ reunión de la Comisión Ballenera Internacional (CBI) (CBI), de la 20 y el 28 de octubre; 2. La Conferencia de las Partes en la Convención Marco de las Naciones Unidas sobre el Cambio Climático (COP22) y la "Proclamación de Acción de Marrakech"; 3. La Conferencia de las Partes (COP 13) del Convenio sobre Diversidad Biológica (CDB): la Declaración de Cancún sobre Integración para la Conservación y Uso Sostenible de la Biodiversidad para el Bienestar; 4. Las decisiones de las triples CoPs: COP 8 Convenio de Estocolmo sobre Contaminantes Orgánicos Persistentes, la Triple COP- COP 13 Convenio de Basilea sobre Residuos Peligrosos y la Triple COP - COP 8 Convenio de Rotterdam sobre consentimiento fundamentado aplicado a ciertos plaguicidas; 5. Las decisiones de la primera Reunión de las Partes del Acuerdo sobre medidas del Estado rector del puerto destinadas a prevenir, desalentar y eliminar la pesca ilegal, no declarada y no reglamentada; 6. La Resolución 71/312 "Nuestros Océanos, nuestro futuro: Llamamiento para la acción” de la Primera Conferencia Mundial de los Océanos; 7. La agenda internacional para los próximos meses.

La crónica jurídica internacional de este nuevo número de la Revista Catalana de Dret Ambiental centra la atención en los eventos de carácter internacional más relevantes de los últimos seis meses, es decir, de octubre del 2016 a julio de 2017 y, en concreto, se abordan los documentos de las diversas conferencias y reuniones de las partes, celebradas en torno a diferentes cuestiones de interés ambiental.

Entre los eventos internacionales más importantes a destacar está: la celebración de la 66 reunión de la Comisión Ballenera Internacional (CBI), de la 20 y el 28 de octubre; en el mes de noviembre fue la celebración de la COP 22 Convenio sobre el Cambio Climático (UNFCCC) de Naciones Unidas en Marrakech; en diciembre, la COP 13 Convenio sobre la Diversidad Biológica (CDB) de Naciones Unidas en México; y en abril-mayo destacar la celebración de la Triple COP-COP 8 Convenio de Estocolmo sobre Contaminantes Orgánicos Persistentes, la Triple COP-COP 13 Convenio de Basilea sobre Residuos Peligrosos y la Triple COP-COP 8 Convenio de Rotterdam sobre consentimiento fundamentado aplicado a ciertos plaguicidas. Finalmente, en mayo también se celebró la primera Reunión de las Partes del Acuerdo sobre medidas del Estado rector del puerto, destinadas a prevenir, desalentar y eliminar la pesca ilegal, no declarada y no reglamentada y en junio, coincidiendo con el día internacional de los océanos, la Conferencia Mundial de los Océanos.

De este período es relevante hacer mención a la adopción de la "Proclamación de Acción de Marrakech" en el ámbito de las negociaciones climáticas y en el ámbito de la protección de la biodiversidad, la adopción de la "Declaración de Cancún”, a través de la cual los más de 140 gobiernos que la han suscrito, se comprometen a integrar la 
biodiversidad estableciendo marcos institucionales y legislativos, e incorporando un enfoque económico, social y cultural inclusivo con pleno respeto por la naturaleza y los derechos humanos.

\section{Las decisiones de $66^{\mathrm{a}}$ reunión de la Comisión Ballenera Internacional (CBI)}

La 66 reunión de la Comisión Ballenera Internacional (CBI) se ha celebrado en Portoroz, en Eslovenia ${ }^{1}$. Esta esta reunión se adoptaron las siguientes resoluciones: la Resolución 2016-1, sobre la mejora de la eficacia de la Comisión Ballenera Internacional; la Resolución 2016-2 sobre Mejorar el Proceso de Revisión de la Caza de Ballenas bajo Permiso Especial; la Resolución 2016-3 sobre los cetáceos y sus contribuciones al funcionamiento de los ecosistemas; la Resolución 2016-4 sobre el Convenio de Minamata; la Resolución 2016-5 sobre la Vaquita en Peligro Crítico fue adoptada por consenso; y la Resolución 2016-6 sobre la creación de un Fondo para fortalecer la capacidad de los gobiernos de medios limitados. Y aunque se discutió una Resolución sobre seguridad alimentaria pero no se llegó a un consenso. Al respecto, Ghana indicó que continuar perfeccionando la propuesta durante el período entre reuniones, lista para ser presentada a la CBI / 67.

En esta reunión, es significativo apuntar que la CBI ha vuelto a rechazar la creación de un santuario ballenero en el Atlántico, lo que significa un segundo rechazo en cuatro años a esta iniciativa que parte de países del hemisferio sur como Argentina, Brasil y Uruguay. El proyecto del Santuario en el Atlántico Sur fue presentado por primera vez en el 2001 por iniciativa de Brasil y con el apoyo de Argentina. El primer intento de aprobación se produjo en la 64 ${ }^{\mathrm{a}}$ reunión de la CBI, celebrada en Panamá del 11 al 15 de junio de 2012. En aquel momento la propuesta latinoamericana recibió el respaldo de 38 de los 61 países presentes en la reunión (es decir, el $65 \%$ de los votos), pero los estatutos de la CBI establecen que las resoluciones deben ser aprobadas por el $75 \%$ de los países del pleno.

La idea de crear un santuario ballenero en el Atlántico consistía en crear un santuario de 20 millones de kilómetros cuadrados para especies de ballenas amenazadas de

\footnotetext{
${ }^{1}$ Para un resumen de las decisiones adoptadas en esta reunión consultar el documento oficial de la CBI disponible

en: https://iwc.int/private/downloads/StI1_9iNdpWlnmS7jUUtXg/RS6360_66_Main_OutcomesV2.pdf (consultado en agosto 2017).
} 
extinción por la caza masiva destinada a la explotación de su carne y su grasa a lo largo del siglo XX. En la actualidad existen dos santuarios en los que está prohibida la caza comercial de ballenas: el del Mar Austral y el del Océano Índico, establecidos por la CBI en 1994 y 1979, respectivamente, y que representan cerca de un tercio de los mares mundiales. Con la aprobación de la propuesta del Santuario del Atlántico Sur se podrían proteger 54 especies que representan más de un $60 \%$ de los cetáceos del planeta, siete de ellas intensamente migratorias.

En esta ocasión, en Eslovenia, la propuesta ha sido rechazada por Japón, Noruega e Islandia, países que practican la caza de ballenas, tuvo 38 votos a favor y 24 en contra, y de nuevo no alcanza el $75 \%$ necesario de los votos emitidos para poder ser adoptada en esta $66^{\circ}$ reunión de la $\mathrm{CBI}$.

Otras de las principales decisiones que se tomaron giran en torno a mejorar la eficacia de la Comisión Ballenera, al proceso de revisión de la caza de ballenas bajo permiso especial, a la creación de un fondo para fortalecer la capacidad de los gobiernos de medios limitados para participar en el trabajo de la Comisión y a proteger la "vaquita" marina, una marsopa que vive del Golfo de California que está en peligro crítico. Adicionalmente se aprobó otra resolución que considera la contribución de los cetáceos para el ecosistema marino.

Aunque la Convención que creó la CBI es para la conservación adecuada de las poblaciones de ballenas y para posibilitar un desarrollo de la industria ballenera de forma ordenada; la mayoría de sus miembros defienden la conservación y uso no letal de los cetáceos. Esta es la posición de varios países latinoamericanos conocidos como el Grupo Buenos Aires, del que forman parte Argentina, Brasil, Chile, Colombia, Costa Rica, Ecuador, Panamá, Perú, México, República Dominicana y Uruguay.

La Comisión, decidió ampliar la colaboración con otras organizaciones como mejor manera de abordar los problemas globales como la captura incidental, los desechos marinos y colisiones entre ballenas y barcos. Durante el encuentro se eligió al japonés Joji Morishita, como presidente de la Comisión Ballenera y Brasil se ofreció a ser la sede del próximo encuentro en 2018.

\section{La Conferencia de las Partes en la Convención Marco de las Naciones Unidas sobre el Cambio Climático (COP22) y la "Proclamación de Acción de Marrakech"}


Del 7 al 18 de noviembre de 2016 tuvo lugar en Bab Ighli, Marrakech (Marruecos) la vigésimo segunda sesión de la Conferencia de las Partes (COP 22), la duodécima sesión de la Conferencia de las Partes en calidad de reunión de las Partes del Protocolo de Kyoto (CMP 12) y la primera sesión de la Conferencia de las Partes del Acuerdo de París $(\mathrm{CMA} 1)^{2}$.

Después de haber logrado la aprobación de un acuerdo universal el año pasado, los desafíos y las exigencias hacia la COP 22 eran altos. Por este motivo, la COP 22 tenía como objetivo precisar las resoluciones tomadas durante la COP 21 para así asegurar la implementación exitosa del Acuerdo de París ${ }^{3}$. En este sentido, la Conferencia puso de manifiesto cómo el mundo está avanzando para la implementación del Acuerdo de París y cómo se mantiene un espíritu constructivo de cooperación multilateral frente al cambio climático.

Durante las negociaciones, los actores acordaron la elaboración de un documento con las reglas bajo las que se regirá el Acuerdo de París y que deberá estar finalizado para 2018. Se prevé que éste mismo asegure la comparabilidad de las Contribuciones Nacionalmente Determinadas (NDCs, por sus siglas en inglés) y el incremento de la ambición de las contribuciones nacionales para mantener la temperatura por debajo de dos grados. Los NDCs son compromisos de mitigación y adaptación al cambio climático, que las partes presentaron ante la Convención Marco de las Naciones Unidas sobre el Cambio Climático (CMNUCC) previamente a la COP21 en París y en las que se definen medidas concretas para alcanzar la meta global de 2 centígrados. En el 2018 estos compromisos estarán sujetos a revisión por primera vez.

Al respecto, los gobiernos han fijado un plazo hasta 2018 para completar las normas de aplicación del Acuerdo de París con el fin de asegurar la confianza, la cooperación y su éxito en los años y décadas venideros.

Durante la COP22 se lanzó la "NDC Partnership" co-presida por Marruecos y Alemania. Esta tiene como objetivo apoyar los países en la implementación de sus NDCs. Para lograr eso, 33 países y 9 instituciones internacionales, entre otros el World Resources Institute,

\footnotetext{
${ }^{2}$ Ver página web de la CoP en: http://www.cop22-morocco.com/ y http://www.cop22.ma/es. (consultadas el 21 de octubre de 2016).

${ }^{3}$ Decisión 1/CP.21, FCCC/CP/2015/L.9, Acuerdo de París, 12 de diciembre 2015. Disponible en línea en: http://unfccc.int/resource/docs/2015/cop21/spa/109s.pdf (última consulta el 2 de octubre 2016).
} 
desarrollarán buenas practicas, herramientas técnicas y modelos de inversiones "climatesmart".

Alemania presentó, como primer país, su estrategia climática a largo plazo. El plan de protección del clima $2050^{4}$ ofrecerá orientación y seguridad para la futura toma de decisiones estratégicas por parte de todos los actores involucrados para que Alemania, hasta mediados del presente siglo, alcance a ser un país neutro en carbono. Poco después, los gobiernos de Estados Unidos, México y Canadá también presentaron sus estrategias climáticas a largo plazo. El objetivo de México $^{5}$ es una reducción de los gases de efecto invernadero del 50\% en 2050 respecto al año 2000. Eso es de suma importancia para la cooperación técnica entre Alemania y México. Al ser de los primeros países en presentar sus estrategias a largo plazo, ambos países refrendan su compromiso y crean un fundamento sólido para una cooperación más estrecha.

El tema de financiación representó otro pilar estratégico de la COP 22. Los países industrializados reafirmaron que van a proporcionar 100,000 millones de dólares para el financiamiento climático. Alemania se comprometió adicionalmente con 50 millones de euros para el fondo de adaptación creado en el marco del protocolo de Kioto. La siguiente cumbre de cambio climático se llevará a cabo en Bonn, Alemania bajo la presidencia de la República Fiji. Eso dará la posibilidad a los pequeños Estados insulares de concientizar sobre su situación, siendo países afectados particularmente por las consecuencias del cambio climático. En la COP, 45 Estados particularmente afectados por el cambio climático, entre ellos muchos pequeños Estados insulares, anunciaron el cambio de su abastecimiento energético a energías renovables.

Esta nueva etapa de implementación y acción para el clima y para el desarrollo sostenible ha quedado plasmada en la llamada "Proclamación de Acción de Marrakech"6, una

\footnotetext{
${ }^{4}$ El Plan de protección del clima, así como un resumen del contenido, se pueden consultar a través del siguiente $\quad$ enlace: http://www.bmub.bund.de/en/topics/climate-energy/climate/detailsclimate/artikel/climate-action-plan-2050-1/. Y el resumen de estrategias a largo plazo, publicados bajo el siguiente enlace: http://unfccc.int/focus/long-term_strategies/items/9971.php. (consultado el 3 de agosto 2017).

5 Documento disponible en: http://unfecc.int/files/focus/long-
term_strategies/application/pdf/mexico_mcs_final_cop22nov16_red.pdf (consultado el 3 de agosto 2017). 6 Documento disponible en: http://unfccc.int/files/meetings/marrakech_nov_2016/application/pdf/marrakech_action_proclamation.pdf
} 
declaración de intenciones que refleja el compromiso mundial para frenar el calentamiento global. El texto recoge la voluntad de todos los asistentes en torno a las acciones que implementarán antes de 2020 y cómo se responderán las necesidades de los países más vulnerables.

El documento también llama a "facilitar el acceso a la financiación para los proyectos climáticos y reforzar las capacidades y esfuerzos de países desarrollados hacia los más pobres para adaptarse a los impactos del calentamiento global”.

Durante la conferencia, empresas, inversores, ciudades y gobiernos locales también han anunciado nuevos compromisos frente al cambio climático que se suman a los miles anunciados en el periodo previo a la conferencia sobre el cambio climático que se celebró en París el año pasado 7 .

En el cierre de la Conferencia se anunció que Fiji ostentará la presidencia de la próxima Conferencia de las Naciones Unidas sobre el cambio climático del 6 al 17 noviembre de 2017 (COP23) que será organizada por la CMNUCC en Bonn ${ }^{8}$.

\section{La Conferencia de las Partes (COP 13) del Convenio sobre Diversidad Biológica (CDB): la Declaración de Cancún sobre Integración para la Conservación y Uso Sostenible de la Biodiversidad para el Bienestar.}

Del 2 al 17 de diciembre de 2016 tuvo lugar la XIII Reunión de la Conferencia de las Partes (COP 13) del Convenio sobre Diversidad Biológica (CDB $)^{9}$, que por primera vez en la historia se celebró, conjuntamente, con la octava COP-MOP del Protocolo de Cartagena (COP-MOP 8) ${ }^{10}$ y la segunda COP-MOP del Protocolo de Nagoya (COP-MOP

(consultado el 3 de agosto 2017).

7 Sobre la agenda de Acción Climática Global, consultar: http://newsroom.unfccc.int/es/accionclimatica/los-actores-no-estatales-se-asocian-con-los-gobiernos-para-impulsar-la-accion-climatica/ (consultado el 3 de agosto 2017).

8 Para conocer los detalles de los resultados obtenidos en la Conferencia de Marrakech, visitar: http://newsroom.unfccc.int/es/noticias/cop22-comunicado-de-prensa-final/ (consultado el 3 de agosto 2017).

${ }^{9}$ Vid. Convenio sobre la diversidad biológica, Río de Janeiro, 5 de junio de 1992 (31 ILM 818, 1993; B.O.E. núm. 27, 1 de febrero de 1994).

${ }^{10}$ Protocolo de Cartagena sobre Seguridad de la Biotecnología, hecho en Montreal el 29 de enero de 2000. 
$2)^{11}$, con el lema "Integrando la biodiversidad para el bienestar". La ciudad mexicana de Cancún, en el estado de Quintana Roo, cerca de diez mil participantes, entre representantes de los países parte, países observadores, organizaciones internacionales y otros interesados se reunieron en Cancún para negociar acuerdos y compromisos para dar impulso a la conservación y uso sostenible de la biodiversidad, así como al cumplimiento del Plan Estratégico para la Diversidad Biológica 2011-2020 y las Metas de Aichi.

La Conferencia de las Naciones Unidas sobre Biodiversidad fue precedida por un Segmento de Alto Nivel los días 2 y 3 de diciembre de 2016. Por primera, vez participaron ministros y representantes de otros sectores distintos al ambiental, quienes sostuvieron diálogos intersectoriales para promover la integración de la conservación y el uso sustentable de la biodiversidad dentro de 4 sectores seleccionados por México. Como resultado de este encuentro, los participantes adoptaron la "Declaración de Cancún sobre Integración de la Conservación y Uso Sostenible de la Biodiversidad para el Bienestar"12, firmada por Ministros y representantes de los sectores ambiental, pero también agrícola, forestal, pesquero y turístico.

Mediante esta Declaración, los países asumen 18 compromisos, entre los más importantes está el de comprometerse a incrementar y fortalecer los sistemas de áreas protegidas ecológicamente; integrar la biodiversidad en los programas educativos para incrementar la conciencia en este tema; y aumentar la cooperación internacional y fomentar la innovación y la transferencia de tecnologías. Asimismo, el documento señala que se facilitará la participación activa y efectiva de todos los actores y sectores y que los países adoptaran medidas para fortalecer las capacidades de los pueblos indígenas y las comunidades locales para la aplicación del Convenio sobre la Diversidad Biológica, mediante el respeto de sus derechos y la utilización sostenible de la biodiversidad. Además, las naciones se comprometen a mejorar el marco normativo de las actividades

BOE Núm: 181 de 30/07/2003 Núm. marginal: 15235.

${ }^{11}$ Vid. Protocolo de Nagoya sobre Acceso y Participación justa y equitativa en los Beneficios que se deriven de su utilización al Convenio sobre la Diversidad Biológica, de 29 de octubre de 2010, Doc. $\mathrm{UNEP} / \mathrm{CBD} / \mathrm{COP} / \mathrm{DEC} / \mathrm{X} / 1$. Para consultar el texto del Protocolo: http://www.cbd.int/cop/cop10/doc/advance-final-unedited-texts/advance-unedited-version-ABS-Protocol-footnote-en.doc. (consultado el 3 de agosto 2017).

12 Para consultar el documento ver: https://www.cbd.int/cop/cop-13/hls/Cancun\%20Declaration-ESPtemporal\%20(NOV-01-2016).pdf (consultado el 3 de agosto 2017). 
del sector privado, optimizar los incentivos y promover herramientas para la conservación y uso sostenible de los recursos naturales.

Sobre la base de esta Declaración, los sectores agrícola, forestal, pesquero y turístico serán sometidos a la integración de la conservación y uso sostenible de la biodiversidad, para que sus actividades productivas y económicas protejan el medio ambiente, mejoren su eficiencia y reduzcan sus emisiones de gases de efecto invernadero.

Además de esta Declaración, en esta COP se adoptaron un total de 72 acuerdos: 37 decisiones para la aplicación del Convenio sobre la Diversidad Biológica (CBD); otras 20 estuvieron enmarcadas en el Protocolo de Cartagena y las 15 restantes con el de Nagoya.

De entre estos acuerdos están los relativos a temas como polinizadores y seguridad alimentaria, protección de ecosistemas y especies en riesgo, contaminación marina y el cambio climático. Asimismo, se tomaron decisiones en aspectos complejos como evaluación de riesgo de los organismos vivos modificados, biología sintética, información de secuencias digitales de recursos genéticos y consentimiento informado previo. También se incluyó la integración de la biodiversidad en los sectores agrícola, pesquero, forestal y turístico, con el fin de alcanzar las metas establecidas en 2010 en Aichi (Japón).

Las Metas de Aichi señalan acciones para proteger y utilizar de manera sostenible la biodiversidad en nuestro planeta. Abordan también cuestiones que van desde la pérdida de los hábitats naturales, la agricultura sostenible y la disminución de las poblaciones de peces, hasta el acceso y el reparto equitativo de los beneficios del uso de los recursos genéticos, el conocimiento indígena y la conciencia de los valores de la biodiversidad. Alcanzar las Metas de Aichi es indispensable para avanzar en los propósitos de otras dos agendas globales históricas acordadas el año pasado: los Objetivos del Desarrollo Sostenible (ODS) y el Acuerdo de París sobre cambio climático

Después de la COP de Cancún, las sedes para las próximas Conferencias de las Partes serán Egipto (COP 14), China (COP 15) y Turquía (COP 16). Además, Perú acogerá las actividades preparatorias rumbo a la COP 15.

\footnotetext{
4. Las decisiones de las triples CoPs: COP 8 Convenio de Estocolmo sobre Contaminantes Orgánicos Persistentes, la Triple COP- COP 13 Convenio de Basilea
} 


\section{sobre Residuos Peligrosos y la Triple COP - COP 8 Convenio de Rotterdam sobre consentimiento fundamentado aplicado a ciertos plaguicidas.}

La octava reunión de la Conferencia de las Partes en el Convenio de Rotterdam ${ }^{13}$ (RC CdP-8) se celebró del 24 de abril al 5 de mayo de 2017 de manera consecutiva con la décima tercera reunión de la Conferencia de las Partes en el Convenio de Basilea ${ }^{14}$ (BC CdP-13) y la octava reunión de la Conferencia de las Partes en el Convenio de Estocolmo ${ }^{15}$ (SC CdP-8) y la temática fue "Un futuro sin tóxicos: Gestión racional de desechos y productos químicos" $"$.

Las Conferencias de las Partes se reunieron en sesiones plenarias para debatir cuestiones relacionadas con la aplicación de los tres Convenios, los cuales establecen un marco internacional de protección de la población y el medio ambiente a través de la gestión racional de químicos, contaminantes orgánicos persistentes y desechos tóxicos. Dentro de los temas que se abordaron destacan: la coordinación internacional entre los tres Convenios, asistencia técnica, recursos y mecanismos financieros y programas de trabajo y cuestiones relacionadas con los presupuestos.

En concreto, en la octava reunión de la Conferencia de las Partes en el Convenio de Rotterdam adoptó 17 decisiones, incluidas seis idénticas para las tres reuniones de la Conferencia de las Partes. Algunos de los principales resultados de la Conferencia contienen la inclusión de cuatro nuevos productos químicos en el anexo III del Convenio, a saber, carbofuran y triclorfón como plaguicidas y parafinas cloradas de cadena corta y compuestos de tributilestaño, como productos químicos industriales, y una decisión para

\footnotetext{
${ }^{13}$ Hecho en Rotterdam (Países Bajos) el 10 de septiembre de 1998. BOE, núm. 73, 25 de marzo de 2004, páginas 12926 a 12937 (12 págs.). Sobre el Convenio, consúltese el sitio oficial: http://www.pic.int/Inicio/tabid/1925/language/es-CO/Default.aspx. (consultado el 3 de agosto 2017).

${ }^{14}$ Convenio de Basilea sobre el control de los movimientos transfronterizos de los desechos peligrosos y su eliminación, Basilea, 22 de marzo de 1989 (BOE, núm. 227, 22 de septiembre de 1994). Sobre el Convenio, consúltese: www.basel.int.

${ }^{15}$ Hecho en Estocolmo el 22 de mayo de 2001. BOE, núm. 151, 23 de junio de 2004, pág. 22737. Sobre el Convenio, consúltese: http://chm.pops.int/.(consultado el 3 de agosto 2017).

${ }^{16}$ Para mayor información sobre las reuniones de las conferencias de las partes en los convenios de Basilea, Estocolmo y Rotterdam, sírvase visitar la página web de Sinergias en: http://synergies.pops.int/Default.aspx?tabid=5306 (consultada el 12 de octubre de 2016).
} 
emprender nuevos trabajos entre períodos de sesiones para mejorar la eficacia del Convenio ${ }^{17}$.

En la decimotercera reunión de la Conferencia de las Partes en el Convenio de Basilea adoptó 24 decisiones, incluidas las seis idénticas para las tres reuniones de la Conferencia de las Partes ${ }^{18}$. Algunos de los principales resultados de la Conferencia fueron la adopción de seis directrices técnicas sobre residuos de COP, la adopción de decisiones sobre el seguimiento de la iniciativa indonesia-suiza dirigida por los países, la Declaración de Cartagena, la presentación de informes nacionales, la asistencia técnica, la creación de soluciones innovadoras para la gestión ambientalmente racional de los residuos domésticos y el programa de trabajo y las actividades del Grupo de Trabajo de composición abierta para 2018-2019, que incluye nuevas áreas de trabajo, como residuos de nanomateriales, plásticos y electrónicos de los documentos de notificación y movimiento.

Finalmente, en el caso de la octava reunión de la Conferencia de las Partes en el Convenio de Estocolmo se adoptaron 27 decisiones, incluidas las seis idénticas para las tres reuniones de la Conferencia de las Partes. Algunos de los principales resultados de la Conferencia incluyen la inclusión de tres nuevos productos químicos recomendados por el POPRC, a saber, el decaBDE y las parafinas cloradas de cadena corta (PCCC) en el anexo A y el hexaclorobutadieno (HCBD) en el anexo C; la evaluación del eficacia de la Convención, lo que dio lugar a medidas en numerosos ámbitos de la Convención para mejorar su aplicación; orientación al mecanismo financiero; una nueva orientación sobre inventarios y MTD y MPA y nuevas medidas para eliminar los $\mathrm{PCB}^{19}$.

\footnotetext{
${ }^{17}$ Consultar Report of the Conference of the Parties to the Rotterdam Convention on the Prior Informed Consent Procedure for Certain Hazardous Chemicals and Pesticides in International Trade on the work of its eighth meeting (advance), UNEP/FAO/RC/COP.8/27.

${ }^{18}$ Incluidas en el documento UNEP / CHW.13/28.Consultar: Report of the Conference of the Parties to the Basel Convention on the Control of Transboundary Movements of Hazardous Wastes and Their Disposal on the work of its thirteenth meeting (advance), UNEP/CHW.13/28.

${ }^{19}$ Consultar: Report of the Conference of the Parties to the Stockholm Convention on Persistent Organic Pollutants on the work of its eighth meeting (advance), UNEP/POPS/COP.8/32.
} 


\section{Las decisiones de la primera Reunión de las Partes del Acuerdo sobre medidas} del Estado rector del puerto destinadas a prevenir, desalentar y eliminar la pesca ilegal, no declarada y no reglamentada

La primera Reunión de las Partes del nuevo tratado internacional de la FAO dirigido a combatir la pesca ilícita se inició el 29 de mayo en Oslo. La pesca ilegal no declarada ni reglamentada se estima en 26 millones de toneladas anuales y cuesta entre 10.000 y 23.000 millones de dólares al sector pesquero en ese mismo periodo de tiempo, según estimaciones de la Organización de la ONU para la Alimentación y la Agricultura (FAO).

El Acuerdo sobre medidas del Estado rector del puerto destinadas a prevenir, desalentar y eliminar la pesca ilegal, no declarada y no reglamentada (acuerdo PSMA, por sus siglas en inglés $)^{20}$, que entró en vigor en junio de 2016, cuenta ahora con 46 partes, siendo una de ellas la que comprende a todos los 28 miembros de la Unión Europea.

El tratado -promovido por la FAO- restringe el acceso portuario a los buques pesqueros que no cumplan con un conjunto de reglas, incluyendo demostrar que tienen licencias apropiadas para operar y que muestren de forma transparente la especie y cantidad de pescado capturado. Representa la piedra angular de años de esfuerzos diplomáticos para combatir la lacra de la pesca ilegal, no declarada y no reglamentada (pesca INDNR), que asciende a 26 millones de toneladas y un valor de 23000 millones de dólares EEUU anuales y representa una gran amenaza en el esfuerzo para impulsar la pesca sostenible en los océanos del mundo.

En esta primera reunión de las partes del PSMA, que se celebra durante una semana, del 29 de mayo al 2 de junio, se espera que defina las responsabilidades de los Estados involucrados, así como de las Organizaciones regionales de gestión pesquera y otros organismos internacionales $^{21}$. La FAO, que ya está muy comprometida en iniciativas de creación de capacidad para apoyar el tratado en todo el mundo, adquiere ahora nuevas obligaciones en relación con la implementación del PSMA, incluyendo el apoyo a una

\footnotetext{
${ }^{20}$ Texto disponible en: http://www.fao.org/fileadmin/user_upload/legal/docs/037t-s.pdf (consultado el 3 de agosto 2017).

${ }^{21}$ Ver: http://www.fao.org/fi/static-media/MeetingDocuments/PSMA/Parties/infls.pdf (consultado el 3 de agosto 2017).
} 
futura reunión de seguimiento, junto con consultas informales eventuales y grupos de trabajo técnicos ad hoc para discutir cuestiones específicas.

También se han abordado las necesidades técnicas de los países en desarrollo, mientras que el primer grupo de trabajo ad hoc ha evaluado los recursos necesarios para garantizar que todos los miembros puedan cumplir sus funciones, incluidos los pequeños Estados insulares en desarrollo (PEID) situados en algunas de las más importantes zonas pesqueras del mundo. En concreto, se invita a las Partes a establecer el Grupo de trabajo ad hoc en virtud de la Parte 6, artículo 21 6) del Acuerdo (en adelante el Grupo de trabajo de la Parte 6), y a examinar y adoptar su Mandato; a abordar las necesidades de los Estados en desarrollo para la aplicación del Acuerdo y determinar las prioridades de asistencia; a instar al Grupo de trabajo de la Parte 6 a examinar el proyecto de Mandato para los mecanismos de financiación que habrá de establecerse en virtud del artículo 21 del Acuerdo que figura en el documento PSMA/2017/Inf.6 ${ }^{22}$.

El PSMA es muy importante para alcanzar el Objetivo de Desarrollo Sostenible 14, que pide de forma explícita que se ponga fin a la pesca INDNR en 2020.

\section{La Resolución 71/312. "Nuestros Océanos, nuestro futuro: Llamamiento para la acción" de la Primera Conferencia Mundial de los Océanos}

La Conferencia Mundial sobre los Océanos ${ }^{23}$, que se celebró del 5 al 9 de junio en Nueva York, fue una reunión de alto nivel de las Naciones Unidas para Apoyar la Consecución del Objetivo de Desarrollo Sostenible 14: Conservar y Utilizar Sosteniblemente los Océanos, los Mares y los Recursos Marinos para el Desarrollo Sostenible, coincidiendo con el Día Mundial de los Océanos. Los Gobiernos de Fiji y de Suecia ejercieron las funciones de anfitriones de la Conferencia.

El objetivo principal de la Conferencia fue adoptar las medidas necesarias para proteger a los mares de acciones humanas como la contaminación, el cambio climático o la sobrepesca. En la cita estuvieron presentes aproximadamente 4.000 delegados, incluyendo 16 jefes de Estado o de Gobierno, los primeros ministros de dos

\footnotetext{
22 Ver el documento de las decisiones en: http://www.fao.org/fi/staticmedia/MeetingDocuments/PSMA/Parties/5s.pdf (consultado el 3 de agosto 2017).

23 Consultar la pàgina oficial de la Conferencia en: http://www.un.org/es/conf/ocean/news.shtml (consultado el 3 de agosto 2017).
} 
adjuntos, 86 ministros, 16 viceministros, otros representantes de gobiernos y organizaciones intergubernamentales, instituciones financieras internacionales $\mathrm{y}$ regionales, la sociedad civil, instituciones académicas y de investigación, pueblos indígenas, comunidades locales, y miembros del sector privado. Ningún representante oficial de los Estados Unidos. La Conferencia se ha organizado en ocho sesiones plenarias y siete diálogos sobre las alianzas, que se celebraron del 5 al 9 de junio de 2017, incluyendo un acto especial para conmemorar el Día Mundial de los Océanos, el 8 de junio de 2017.

Esta Conferencia se ha celebrado bajo la premisa de que la salud del planeta también depende, de la calidad del agua marina. La influencia de los océanos es fundamental para la vida humana, pero en las dos últimas décadas estos han sufrido un alarmante proceso de degradación. Los 193 Estados Miembros de las Naciones Unidas acordaron unánimemente establecer una serie de medidas que comenzarán a revertir el declive de la salud de los océanos.

Por este motivo, el principal resultado de la Conferencia es la adopción de la Resolución "Nuestros Oceános, nuestro futuro: Llamamiento para la Acción”24. El documento final, con más de 1.300 acciones a realizar, marcará el comienzo de la gestión y conservación del océano.

Así, el documento aprobado incluye la voluntad de abordar la consecución del Objetivo 14 de manera integrada y coordinada y promover políticas y medidas al respecto; también la implementación de estrategias para proteger y gestionar de forma sostenible la pesca, restablecer las poblaciones de peces en peligro en el menor tiempo posible y la prohibición de aquellas subvenciones que contribuyen a la pesca ilegal, no declarada y no reglamentada; apoyar los planes orientados a fomentar la educación relacionada con los océanos; acelerar las medidas para prevenir y reducir significativamente todo tipo de contaminación marina, en particular la resultante de actividades realizadas en tierra; promover la prevención y la minimización de los desechos, fomentar las modalidades de consumo y producción sostenibles; apoyar la utilización de mecanismos de gestión eficaces y adecuados basados en zonas geográficas, incluidas las zonas marinas protegides; elaborar y aplicar medidas eficaces de adaptación y mitigación; actuar con

\footnotetext{
${ }^{24}$ Resolución aprobada por la Asamblea General el 6 de julio de 2017 [sin remisión previa a una Comisión Principal (A/71/L.74)] 71/312. Nuestros océanos, nuestro futuro: llamamiento a la acción. Texto disponible en: http://www.un.org/ga/search/view_doc.asp?symbol=A/RES/71/312\&Lang=S
} 
decisión para prohibir ciertas formas de subvenciones a la pesca que contribuyen a la sobrecapacidad y la pesca excesiva, entre otras acciones.

En este sentido, algunos países se comprometieron a declarar zonas restringidas a ciertas formas de pesca y a adoptar medidas para proteger los ecosistemas costeros y de carbono azul, como manglares, marismas, praderas marinas y arrecifes de coral.

El documento también aborda los efectos adversos del cambio climático en los océanos, como el aumento de la temperatura y del nivel del mar, la acidificación, la erosión de zonas costeras y la disminución de la cobertura del hielo polar. Al respecto, Naciones Unidas señaló la importancia de esta Conferencia en el proceso de aplicación del Acuerdo de París sobre el cambio climático.

Todas estas propuestas de actuación se adoptaron formalmente al finalizar la conferencia, así como los informes de los diálogos de siete socios que se centraron en ampliar las soluciones y los compromisos de actuación.

Y a pesar de que días antes de adoptarse esta Resolución, EE. UU. anunciaba su retirada del Acuerdo de París, sin duda, este documento representa un paso adelante, por parte de la sociedad internacional, en la consecución del Objetivo 14 en el contexto de la implementación de la Agenda 2030.

\section{La agenda internacional para los próximos meses}

Para finalizar la presente crónica internacional, se da noticia de la agenda internacional, con la relación de las principales reuniones internacionales que se celebrarán en los próximos meses, es decir de julio a octubre de 2017.

En septiembre, está prevista la celebración de la primera reunión de la Conferencia de las Partes en el Convenio de Minamata sobre el Mercurio (COP1), que tendrá lugar, en concreto, del 24 al 29 de septiembre de 2017 en el Centro Internacional de Conferencias de Ginebra, Suiza.

El siguiente mes, se celebrará la $12^{\text {a }}$ sesión de la Conferencia de las partes de la Convención sobre la Conservación de las Especies Migratorias de Animales Silvestres (CMS COP 12), así como las reuniones asociadas a la misma tendrán lugar entre los días 22 y 28 octubre 2017 en Manila, Filipinas. 
A finales de año, se celebrará la Conferencia de Naciones Unidas sobre Cambio Climático (COP23) en Bonn, bajo la presidencia de las islas Fiji. Aunque Fiji presidirá la COP23 en 2017, la CoP se realizará en Bonn, sede del secretariado ejecutivo de la Convención de Naciones Unidas sobre Cambio Climático (UNFCCC). Esta reunión, como la celebrada en Marrakech, se centrará en las cuestiones de aplicación del Acuerdo de París, en vigor desde el 4 de noviembre de 2016.

Estas son solo algunas de las citas internacionales más relevantes ${ }^{25}$ que van a producirse en los próximos meses y que sin duda van a ser objeto de atención y análisis en la siguiente entrega de la crónica internacional, correspondiente al segundo semestre, de la Revista Catalana de Dret Ambiental.

25 Pueden seguir un calendario más detallado de estos eventos internacionales en: http://www.unep.org/ecalendar/ y también en http://sd.iisd.org/events/ (consulta realizada el 24 de octubre de 2016). 